Volume 9

Issue 1 The Edge of the Known is Infinite:

Original Contributions in Undergraduate

Research

\title{
Characterization of the Role that Alternative Ribonucleotide Reductases Play in Restoring Replication in the Presence of Hydroxyurea in Escherichia Coli
}

Michael Sadek

Portland State University

Follow this and additional works at: https://pdxscholar.library.pdx.edu/mcnair

Part of the Amino Acids, Peptides, and Proteins Commons, Biological Factors Commons, Cell Biology Commons, and the Chemical Actions and Uses Commons

Let us know how access to this document benefits you.

\section{Recommended Citation}

Sadek, Michael (2015) "Characterization of the Role that Alternative Ribonucleotide Reductases Play in Restoring Replication in the Presence of Hydroxyurea in Escherichia Coli," PSU McNair Scholars Online Journal: Vol. 9: Iss. 1, Article 8.

https://doi.org/10.15760/mcnair.2015.111

This open access Article is distributed under the terms of the Creative Commons Attribution-NonCommercialShareAlike 4.0 International License (CC BY-NC-SA 4.0). All documents in PDXScholar should meet accessibility standards. If we can make this document more accessible to you, contact our team. 
Portland State University McNair Research Journal 2015

Characterization of the role that alternative ribonucleotide reductases play in restoring replication in the presence of hydroxyurea in Escherichia coli

\author{
By \\ Michael Sadek
}

Faculty Mentor:

Dr. Justin Courcelle

Citation: Sadek, M. Characterization of the role that alternative ribonucleotide reductases play in restoring replication in the presence of hydroxyurea in Escherichia coli. Portland State University McNair Scholars Online Journal, Vol. 9, 2015. 


\begin{abstract}
DNA replication is essential for cells to grow and divide. Ribonucleotide reductase is an essential enzyme that is responsible for the formation of deoxyribonucleotides that are used in DNA synthesis during replication. Hydroxyurea is a chemotherapeutic agent that is thought to work by specifically inhibiting the ribonuceotide reductase to prevent replication. However, recent studies in E. coli have shown that following an initial period of inhibition, DNA synthesis then recovers in the presence of hydroxyurea, suggesting that the mode of death and cellular response to hydroxyurea is more complex than originally proposed. The E.coli genome encodes three ribonucleotide reductases that are thought to operate under distinct cellular conditions. Here, we examined whether either of the two alternative riboucleotide reductases may be resistant to hydroxyurea treatment and allow replication to recover in its presence. To test this, mutants lacking either the iron-starvation reductase, NrdEF, or the anaerobic reductase, NrdDG were examined for their ability to recover DNA synthesis in the presence of hydroxyurea. We observed that, both $n r d E F$ and $n r d D G$ mutants recovered replication similar to wild type cultures, suggesting that these enzymes are not responsible for the observed recovery. We discuss these results in relation to their implications for the mechanism by which hydroxyurea operates to inhibit and kill growing cells.
\end{abstract}




\section{Introductions}

Replication is the process by which the cell duplicates its DNA. Cells use deoxyribonucleotide triphosphates to build DNA. Ribonucleotide reductase is the enzyme that is responsible for the reduction of ribonucleotides triphosphates to form deoxyribonucleotides-triphosphates that are precursors of DNA synthesis (1). This enzyme plays a critical role in regulating the rate of DNA synthesis as well as maintaining the fidelity of replication (1). The primary ribonucleotide reductase found in aerobic bacteria and mammals consists of two non-identical subunits, protein R1 and R2. These proteins need $\mathrm{Mg}^{+2}$ in order to be active (2). The R1 protein contains the redox-active thiols in each of its two active sites. The R2 subunit contains a binuclear iron center and organic free radical (3).

Escherichia coli contain three ribonucleotide reductases that each function under different growth conditions. The class la enzyme, encoded by $n r d A B$, consists of two subunits. The larger subunit is encoded by $n r d A$ and contains the active site and allosteric binding site. The smaller subunit is encoded by $n r d B$ and contains a dinuclear iron center (4). NrdAB is an iron dependant enzyme and is essential for aerobic growth. The class $1 \mathrm{~b}$ enzyme is encoded by $n r d E F$, utilizes manganese rather than iron. The alternative aerobic deoxyribonucleotide reductase of $E$. coli, NrdEF, is a manganese-dependent enzyme that enables cell replication during periods of iron starvation (5). The E. coli genome has also $n r d D$ and $n r d G$ genes that are essential for strict anaerobic growth (6).

Hydroxyurea is a therapeutic agent extensively used in the clinical treatment of hematological malignancies $(7,8)$, chronic myelogenous leukemia (9), sickle-cell disease (10), carcinomas, and other diseases (7). Hydroxyurea is thought to specifically inhibit ribonucleotide reductase, depleting the pools of deoxyribonucleoside triphosphates, thereby leading to stalled replication forks that prevent cells from progressing through S phase (1). Mechanistically, hydroxyurea diffuses into cells and forms a free radical nitroxide, quenching the tyrosyl free radical at the active site of the $\mathrm{M} 2$ protein subunit of ribonucleotide reductase and inactivating the enzyme (11).

Although hydroxyurea has been used to study replication fork arrest for decades, a number of mechanic questions remain about how it affects replication, dNTP pools, and cell viability. In addition to pausing replication, some evidence suggests that hydroxyurea also induces DNA damage (12), which may contribute to its effectiveness in chemotherapies. Treatment with hydroxyurea leads to loss of cell viability and eventual cell lyses (13), an observation that is inconsistent with hydroxyurea simply pausing replication forks (14). The cell lysis correlates with an increase in oxidative free radical formation, suggesting that oxidative damage to DNA and potentially proteins plays a role in the observed lethality. Intriguingly, prolonged hydroxyurea stress activates mazEF and relBE toxin/anti-toxin modules that have been implicated in cell lysis following either oxidative damage or depletion of thymine pools in a phenomenon termed "thymineless" death (15). These observations implicate oxidative or free radical damage as a potential mechanism for hydroxyurea-mediated lethality.

In addition, there are conflicting reports in the literature as to the effect of hydroxyurea on replication and deoxyribonucleotide pools in vivo (1). Some studies found that hydroxyurea arrests DNA replication by a mechanism that preserves basal dNTP pools (16). And a recent 
study from our lab by Neda Savic demonstrated that replication appears to recover in E. coli treated with hydroxyurea after a brief inhibition period (17). The mechanism by which replication recovers under these conditions is not presently understood, however it may be that other ribonucleotide reductases in E.coli are less sensitive to the inhibitory effects of hydroxyurea, or become activated when free radical stress conditions are induced by hydroxyurea treatment.

To further understand the mechanism by which replication is inhibited but then recovers when hydroxyurea is present, I examined how the rate of DNA synthesis is affected by hydroxyurea in ribonucleotide reductase mutants. To accomplish this, I genetically engineered mutants lacking either NrdEF or NrdDG and then tested whether the absence of these reductases affected its ability to recover DNA synthesis in the presence of hydroxyurea. I observed that both mutants recovered replication similar to wild type cultures.

\section{Material and Methods}

Bacterial Strains, and Culture Medium

The SR108 parental strain is a thymine autotrophic (thyA36 deoC2) derivative of W3110 (18). All bacterial strains were propagated in Davis medium (19) supplemented with $0.4 \%$ glucose and $0.2 \%$ CAS amino acids (DGC). DGC growth medium for thymine auxotroph was also supplemented with $10 \mu \mathrm{g} / \mathrm{ml}$ thymine (DGCthy).

Construction of Escherichia coli deletion mutants by $P 1$ transduction Isogenic mutants deleted for either $n r d E F$ or $n r d D$ or $n r d G$ were constructed using phage P1 to transduce the genes from donor strains CL2581 containing the nrdEF::cat allele (Courcelle Lab Stock), JW4196-3 containing the del(nrdG784::kan) allele (20) and JW4197-1 containing the del(nrdD785::kan) allele into SR108 recipient (21).

The strain donors that had the gene of interest were streaked on agar plates overnight. Then a single colony of each strain was grown in $5 \mathrm{ml}$ Luria Butani (LB) media (19) supplemented with $5 \mathrm{mM} \mathrm{CaCl} 2$ overnight. $100 \mu \mathrm{L}, 10 \mu \mathrm{L}$ and $1 \mu \mathrm{L}$ of P1 virion lysate was added to three $1 \mathrm{ml}$ aliquots of the overnight culture. The mixtures were then incubated in a $37 \mathrm{C}$ water bath for one hour.

While the infection was taking place, $R$ top-agar was melted and kept at $42 \mathrm{C}$ beside the $\mathrm{LB}$ agar plates. Then at the end of incubation, $4 \mathrm{ml}$ top agar was added to each tube and the mix was poured on LB plate. Plates were then incubated overnight at 37C. The agar, containing the lysate was removed, collected in tubes, and centrifuged for 15 minutes at 14,000 rpm. The supernatant was collected into fresh tubes. $100 \mu \mathrm{l}$ of chloroform was added, and the lysate was centrifuged again for 15 minutes. The supernatant containing the phage was collected and a few drops of chloroform were added to it before it was stored at $4 \mathrm{C}$.

For transduction, the recipient strain, SR108, was cultured and grown in LBthy media supplemented with $5 \mathrm{~mm} \mathrm{CaCl} 2$ at $37 \mathrm{C}$ overnight. $100 \mu \mathrm{l}$ of $\mathrm{P} 1$ phage lysate made from the donor strains was added to 100 ul of culture, and incubated for 15 minutes at $30 \mathrm{C}$ to allow infection to occur. At the end of incubation, $40 \mu \mathrm{l} 1 \mathrm{M}$ of sodium citrate was added to each tube. 
The mixture was then spread on LB plates containing the appropriate antibiotic for selection, and plates were incubated overnight at $37 \mathrm{C}$.

Replication Inhibition Assays

Sample cultures were prepared for the three mutants and the wild type. $0.4 \mathrm{ml}$ of overnight culture was diluted into $40 \mathrm{ml}$ DGCthy containing $0.1 \mathrm{uCi} / \mathrm{ml} 14 \mathrm{C}$-thymine and it was incubated in $37 \mathrm{C}$ water bath for three hours. A label mix was prepared by adding $10 \mu \mathrm{Ci} / \mathrm{ml}$ of $3 \mathrm{H}$ thymidine to $2 \mathrm{ml}$ DGCthy media. $0.5 \mathrm{ml}$ of the labeling mix was added to the PP tubes. 2-0.5 $\mathrm{ml}$ aliquots per treatment were placed into the tubes containing labeling mix. They were vortexed for 2 seconds and returned to $37 \mathrm{C}$ water bath for 2 minutes. Cultures were split into 2-20 ml aliquots. Then $1 \mathrm{ml}$ of $\mathrm{H} 2 \mathrm{O}$ was added to one flask and hydroxyurea to the other one. Aliquots were pulsed and collected in the same way at $-5,0,5,10,20,30,40,50$ and 60 minutes. Cells were then lysed with $5 \%$ cold trichloroacetic acid (TCA, Fisher brand) and the precipitated DNA was collected on glass fiber filters (Millipore). The filters were rinsed twice with ethanol, dried, and then the amount of $3 \mathrm{H}$ and $14 \mathrm{C}$ on each filter was determined using a scintillation counte

\section{Results}

To address how the alternative ribonucleotide reductase affects the recovery of replication in the presence of hydroxyurea, isogenic mutants were constructed containing deletions in $n r d E F, n r d D$ and $n r d G$. To monitor the rate of DNA synthesis and overall DNA accumulation that occurred in the presence of hydroxyurea, the amount of $3 \mathrm{H}$ thymidine incorporated during a 2 min pulse into cultures pre-labeled with 14C-thymine was followed over time. To this end, growing $14 \mathrm{C}$-thymine prelabeled cultures were split and either treated with $100 \mathrm{mM}$ hydroxyurea, or mock-treated. Then, aliquots of the culture were pulse labeled for $2 \mathrm{~min}$ at various time points, and the amount of $3 \mathrm{H}$ (rate of synthesis) and $14 \mathrm{C}$ (total DNA accumulation) incorporated into the DNA at each time point was determined. Consistent with previous observations from our lab (21); following addition of hydroxyurea in wild type cultures, the rate of replication was inhibited by more than $90 \%$ within 5 minutes of hydroxyurea addition Figure 1. However, the rate of replication then began to recover within 10 minutes of treatment, and had fully recovered to its pretreated rate by the end of the 60 minute time course. The overall accumulation of DNA was found to be less after adding hydroxyurea. I observed that total DNA accumulation was less than untreated cultures by the end of the 60 minute time course.

In mock treated cultures, the rate of DNA continued at a similar rate throughout the time course and DNA continued to accumulate. 

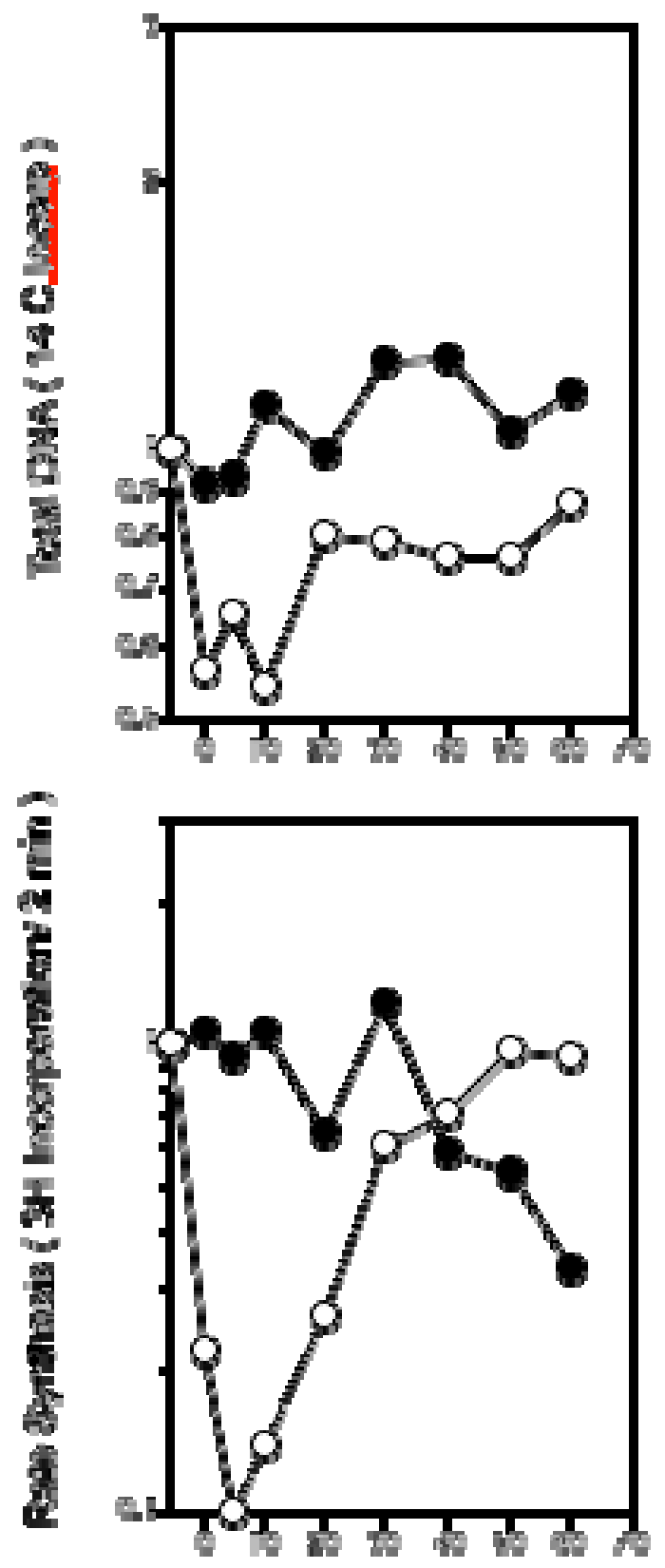

Ime ựini
Figure (1): Following an initial period of inhibition, the rate of replication recovers in the presence of hydroxyurea. Cultures grown in media supplemented with 14C-thymine were labeled with a pulse of $3 \mathrm{H}$-thymidine for $2 \mathrm{~min}$ at various time points following the addition of hydroxyurea. The amounts of $3 \mathrm{H}$ and 14C incorporated into the DNA (relative to pre treatment levels) are plotted over time. (Top) DNA accumulation; black circles, mock treatment; white circles, 100mM HU.(Bottom) Rate of synthesis; black circles, mock treatment; white circles, $100 \mathrm{mM} \mathrm{HU}$. The initial amount of $3 \mathrm{H}$ and $14 \mathrm{C}$ at 5 minutes prior to treatment were 13935CPM and 3056.5CPM respectively. 
To examine whether the Mn-dependent, cryptic ribonucleotide reductase, encoded by nrdEF, was responsible for the observed recovery of replication; I examined how replication was affected in hydroxyurea-treated cultures of $n r d E F$ mutants. I observed that the inhibition and recovery looked similar to that in wild type cultures. As shown in Figure 2, the rate of replication was inhibited by $90 \%$ within 5 minutes of addition. However, the rate of replication then began to recover, reaching rates similar to those in the mock treated samples by the end of the time course. The overall accumulation of DNA was found to be less in the presence of hydroxyurea. I found that DNA accumulation in the treated sample was inhibited relative to the mock treated culture.

I interpret these results to indicate that NrdEF is not required for replication to recover in the presence of hydroxyurea. 


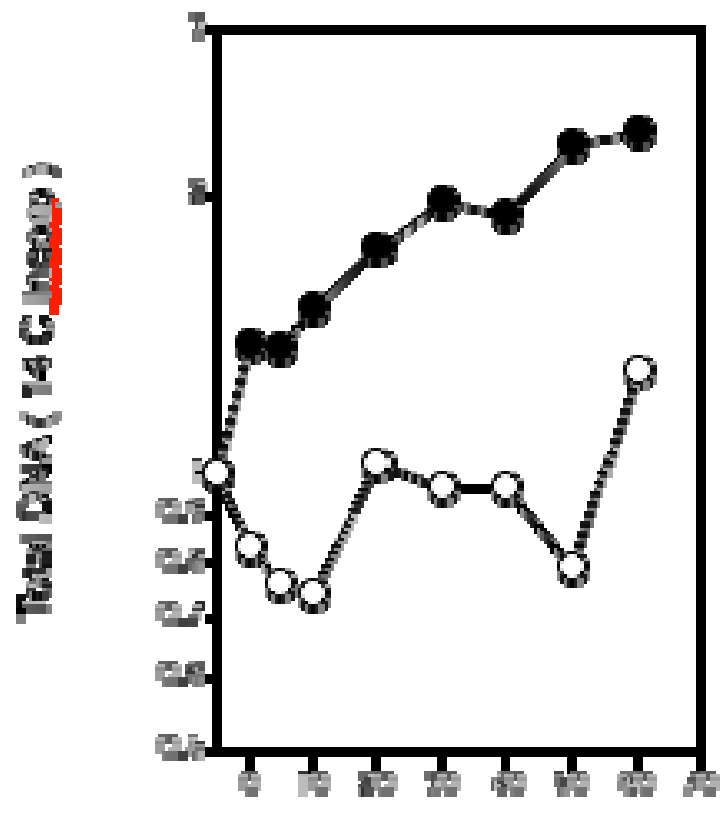

Figure (2): The absence of NrdEF does not prevent replication from recovering in the presence of hydroxyurea. Cultures grown in media supplemented with 14C-thymine were labeled with a pulse of $3 \mathrm{H}$-thymidine for $2 \mathrm{~min}$ at various time points following the addition of hydroxyurea. The amounts of $3 \mathrm{H}$ and $14 \mathrm{C}$ incorporated into the DNA (relative to pre treatment levels) are plotted over time. (Top) DNA accumulation; black circles, mock treatment; white circles, 100mM HU. (Bottom) Rate of Synthesis; black circles, mock treatment; white circles, $100 \mathrm{mM} \mathrm{HU}$. The initial amount of $3 \mathrm{H}$ and $14 \mathrm{C}$ at 5 minutes prior to treatment were $8800 \mathrm{CPM}$ and 1752.5 CPM respectively.

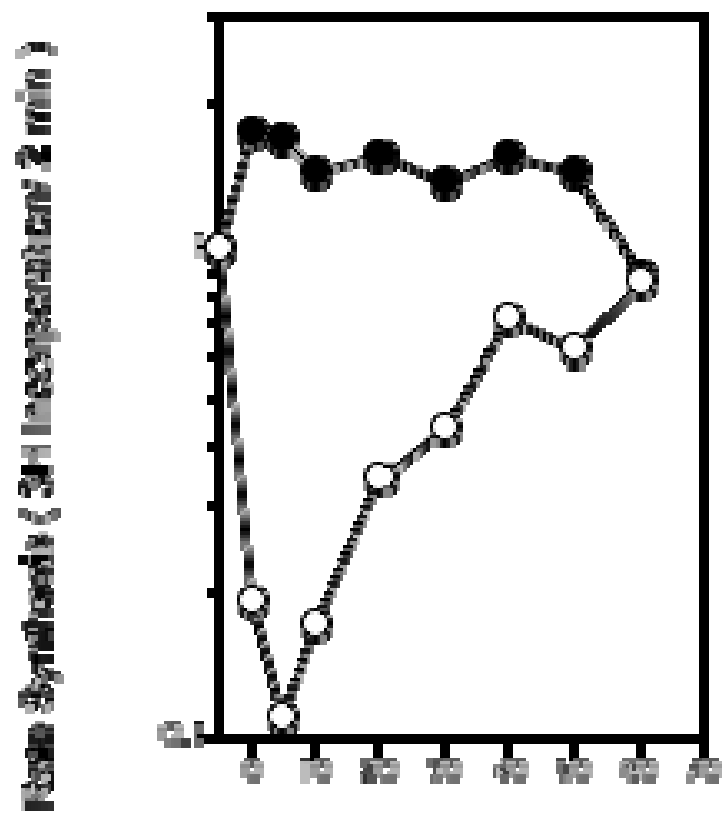

Trra ửning 
I next examined whether the anaerobic ribonucleotide reductase, encoded by $n r d D$ and $n r d G$, affected the ability of cells to restore replication. When I examined replication in hydroxyureatreated cultures of $n r d D$ mutants, I observed that the inhibition and recovery looked similar to that in treated wild type cultures. As shown in Figure 3A, the rate of replication was inhibited by more than $70 \%$ within 5 minutes of addition. However, the rate of replication then began to recover, reaching a rate similar to that of the mock treated culture by the end of the time course. The overall accumulation of DNA was also inhibited in the hydroxyurea-treated $n r d D$ mutant to an extent similar to that seen in treated wild type culture.

Similarly, when I examined replication in hydroxyurea-treated cultures of nrdG mutants, I observed that the inhibition and recovery looked similar to that in wild type cultures. As shown in Figure 3B, the rate of replication was inhibited by $90 \%$ within 5 minutes of addition. However, similar to wild type cultures, the rate of replication recovered to near pretreatment levels by the end of the 60 minute time course. Also, similar to the other strains examined, the overall accumulation of DNA was partially inhibited by the addition of hydroxyurea.

Taken together, these results indicate that neither that NrdD nor NrdG is required for replication to recover from hydroxyurea. 
A)
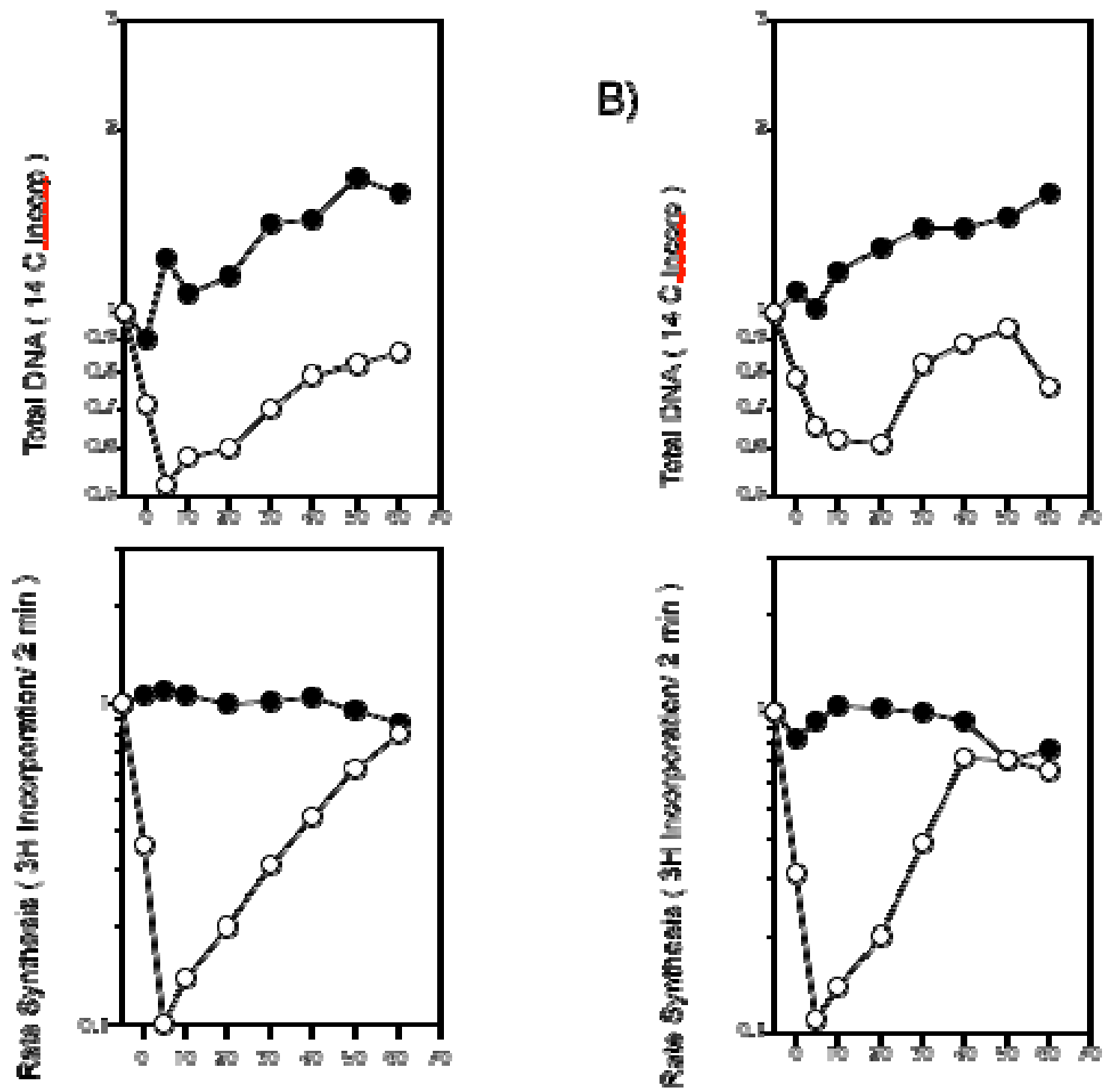

Trra ửrinị

Trro ựinin

Figure (3): The absence of (A) NrdD or (B) NrdG does not prevent replication from recovering in the presence of hydroxyurea. . Cultures grown in media supplemented with 14C-thymine were labeled with a pulse of $3 \mathrm{H}$-thymidine for $2 \mathrm{~min}$ at various time points following the addition of hydroxyurea. The amounts of $3 \mathrm{H}$ and $14 \mathrm{C}$ incorporated into the DNA (relative to pre treatment levels) are plotted over time. (Top) DNA accumulation; black circles, mock treatment; white circles, 100mM HU. (Bottom) Rate of Synthesis; black circles, mock treatment; white circles, $100 \mathrm{mM} \mathrm{HU}$. The initial amount of $3 \mathrm{H}$ and $14 \mathrm{C}$ at 5 minutes prior to treatment were 25536.5CPM and 3021CPM respectively in nrdD mutant. The initial amount of $3 \mathrm{H}$ and $14 \mathrm{C}$ at 5 minutes prior to treatment were $32304.5 \mathrm{CPM}$ and 3506.5 CPM respectively in nrdG mutant. 


\section{Discussion}

The recovery of DNA synthesis after adding hydroxyurea in each mutant was unexpected as hydroxyurea is thought to inhibit the ribonucleotide reduction process which is essential for the replication to continue. Recovery of DNA synthesis after adding hydroxyurea in each mutant showed that neither the cryptic, NrdEF, or anaerobic $N r d D G$ reductases were responsible for this recovery. From these results, I hypothesize that there is another mechanism that was responsible for this recovery. One possibility is that replication in $E$. coli can resume despite reduced concentrations of dNTPs following hydroxyurea treatment. In future studies, it would be useful to follow the ribonucleotide pools over time in the presence of hydroxyurea to directly determine if the dNTP levels also recover, similar to replication.

A second possibility is that dNTP salvage pathways are up regulated. E.coli contains several pathways for importing dNTPs from the media which may allow some replication to recover (21). One could address this by looking at mutants having the salvage pathways inactivated, to see if replication is still able to recover in the presence of hydroxyurea.

A final possibility is that some mechanism exists by which the primary ribonucleotide reductase becomes resistant to hydroxyurea during periods of chronic exposure. Again, one could address this possibility by monitoring the dNTP pool levels during hydroxyurea treatment.

In this study, we showed that the recovery of DNA synthesis in the presence of hydroxyurea is not due to the alternative ribonucleotide reductases, NrdEF or NrdDG. 


\section{References}

Krakoff, I. H., Brown, N. C. \& Reichard, P. Inhibition of ribonucleoside diphosphate reductase by hydroxyurea. Cancer Res. 28, 1559-1565 (1968).

Brown, N. C. \& Reichard, P. Ribonucleoside diphosphate reductase. Formation of active and inactive complexes of proteins B1 and B2. J. Mol. Biol. 46, 25-38 (1969). http://dx.doi.org/10.1016/0022-2836(69)90055-2

Stubbe, J. Di-iron-tyrosyl radical ribonucleotide reductases. Curr Opin Chem Biol 7, 183-188 (2003). http://dx.doi.org/10.1016/S1367-5931(03)00025-5

Nordlund, P., Sjöberg, B. M. \& Eklund, H. Three-dimensional structure of the free radical protein of ribonucleotide reductase. Nature 345, 593-598 (1990).

http://dx.doi.org/10.1038/345593a0

Martin, J. E. \& Imlay, J. A. The alternative aerobic ribonucleotide reductase of Escherichia coli, $\mathrm{NrdEF}$, is a manganese-dependent enzyme that enables cell replication during periods of iron starvation.Mol. Microbiol. 80, 319-334 (2011). http://dx.doi.org/10.1111/i.1365-

2958.2011.07593.x

Garriga, X. et al. nrdDandnrdGGenes Are Essential for Strict Anaerobic Growth of Escherichia coli. Biochemical and Biophysical Research Communications 229, 189-192 (1996).

http://dx.doi.org/10.1006/bbrc.1996.1778

Stearns, B., Losee, K. A. \& Bernstein, J. HYDROXYUREA. A NEW TYPE OF POTENTIAL ANTITUMOR AGENT. J. Med. Chem. 6, 201 (1963). http://dx.doi.org/10.1021/jm00338a026

Stauber, R. H. et al. A combination of a ribonucleotide reductase inhibitor and histone deacetylase inhibitors down regulates EGFR and triggers BIM-dependent apoptosis in head and neck cancer.Oncotarget 3, 31-43 (2011).

Piccinini, G, A Foli et al. "Complementary Antiviral Efficacy of Hydroxyurea and Protease Inhibitors in Human Immunodeficiency Virus-Infected Dendritic Cells and Lymphocytes." J Virol 76.5 (2002): 2274-78. http://dx.doi.org/10.1128/jvi.76.5.2274-2278.2002

Segal, J. B. et al. Hydroxyurea for the Treatment of Sickle Cell Disease. (Agency for Healthcare Research and Quality (US), 2008).

Yarbro, J. W. Mechanism of action of hydroxyurea. Seminars in oncology 19, 1-10 (1992).

Sakano, K., Oikawa, S., Hasegawa, K. \& Kawanishi, S. Hydroxyurea induces site-specific DNA damage via formation of hydrogen peroxide and nitric oxide. Jpn. J. Cancer Res. 92, 11661174 (2001). http://dx.doi.org/10.1111/j.1349-7006.2001.tb02136.x 
Davies, B. W. et al. Hydroxyurea induces hydroxyl radical-mediated cell death in Escherichia coli. Mol Cell 36, 845-860 (2009). http://dx.doi.org/10.1016/j.molcel.2009.11.024

Timson, J. Hydroxyurea. Mutat. Res. 32, 115-132 (1975). http://dx.doi.org/10.1016/0165$\underline{1110(75) 90002-0}$

Young, C. W., Schochetman, G., Hodas, S. \& Balis, M. E. Inhibition of DNA synthesis by hydroxyurea: structure-activity relationships. Cancer Res. 27, 535-540 (1967).

Koç, A., Wheeler, L. J., Mathews, C. K. \& Merrill, G. F. Hydroxyurea arrests DNA replication by a mechanism that preserves basal dNTP pools. J. Biol. Chem. 279, 223-230 (2004).

http://dx.doi.org/10.1074/jbc.M303952200

Savic, Neda. "Characterization of the Replication Rate and Intermediates Produced Following Hydroxyurea Treatment in Escherichia Coli." Portland State University. University Honors Thesis, 2012.

Mellon, I. \& Hanawalt, P. C. Induction of the Escherichia coli lactose operon selectively increases repair of its transcribed DNA strand. Nature 342, 95-98 (1989). http://dx.doi.org/10.1038/342095a0

Davis, BD. The Isolation of Biochemically Deficient Mutants of Bacteria by Means of Penicillin. Proc Natl Acad Sci USA 35, 1-10 (1949). http://dx.doi.org/10.1073/pnas.35.1.1

Baba, T. et al. Construction of Escherichia coli K-12 in-frame, single-gene knockout mutants: the Keio collection. Mol SystBiol 2, 2006.0008 (2006).

Schaaper, R. M. \& Mathews, C. K. Mutational consequences of dNTP pool imbalances in E. coli. DNA Repair (Amst) 12, 73-79 (2013). http://dx.doi.org/10.1016/i.dnarep.2012.10.011 